\title{
Assessment of Intraoral Findings of Neonates, Born in and around Meerut City
}

\author{
Madan M Niranjan ${ }^{1}$, Nikhil Srivastava ${ }^{2}$, Vivek Rana ${ }^{3}$, Preetika Chandna ${ }^{4}$
}

\begin{abstract}
Aim: The aim of this study is to evaluate the occlusal relationships between the gum pads of neonates and to record intraoral findings in neonates and compare with the existing findings.

Materials and methods: The oral cavities of 1,000 healthy neonates from birth to 1 month of age were examined to evaluate the occlusal relationships between the gum pads and to record other intraoral findings in neonates and compare with the existing findings. Oral findings recorded were oral mucosal cysts, Fordyce's spot, Epstein's pearls, Bohn's nodules, ankyloglossia, natal or neonatal teeth and attachment of the upper midline frenum. Relationship between the alveolar ridges was also recorded. Obtained data were evaluated and statistically analyzed using the Statistical Package for Social Sciences (SPSS) version 21.0 and Epi-info version 3.0.

Results: The maxillo-mandibular relationship of gum pads in approximately $97 \%$ cases, the mandible was found distal and lingual to maxilla. The presence of anterior open bite was observed in $9.1 \%$ cases. The most common intraoral finding in examined neonates was an oral mucosal cyst, $61.2 \%$. The presence of Epstein's pearls was the next most common finding 38.3\%. Fordyce's spots were found only in $1.2 \%$ of cases, while the presence of natal/neonatal teeth was only in $0.6 \%$ of cases. Ankyloglossia was present in only $0.5 \%$ neonates. Maxillary frenum was present in $79.1 \%$ of cases and it was attached on the alveolar crest.
\end{abstract}

Conclusion: The results of the present study were in accordance with the pre-existing data. However, anterior open-bite was seen in significantly less percentage of the population.

Keywords: Anterior open bite, Epstein's pearls, Fordyce's spots, Maxilla-mandibular relationship, Gum pad relationship, Oral mucosal cyst. International Journal of Clinical Pediatric Dentistry (2020): 10.5005/jp-journals-10005-1584

\section{INTRODUCTION}

An infant's mouth is unique presenting many features that are peculiar. ${ }^{1}$ A peculiar feature in infants is the occlusal relationship between the gum pads. Clinch classified the relationship of the gum pads into three categories: ${ }^{2}$ (1) mandibular arch positioned slightly lingual to the maxillary arch in both the incisor and the molar region (70\%); (2) mandibular arch positioned slightly lingual and distal to the maxillary arch in the molar region, but definitely distal in the incisor region (27\%); (3) mandibular arch positioned definitely distal in both the molar and the incisor regions (3\%).

The antero-posterior relationship may be classified as a distal, edge-to-edge, or mesial position of the anterior segment of the mandibular ridge in relation to that of the maxillary ridge. The buccolingual relationship may be classified as lingual-edge-toedge or buccal position of the mandibular ridges in relation to the maxillary. ${ }^{3}$ The maxillary and mandibular gum pads have frequently been illustrated by describing a vertical opening in the anterior area, with only posterior segments touching. More often, the maxillary gum demonstrates a slight overlap in relation to the mandibular gum pad, both horizontally and vertically, thereby providing a more efficient way of squeezing milk during feeding. ${ }^{4}$

A variety of anomalies are found in the oral cavity of the neonates. From classified conditions affecting newborns as Epstein's pearls, Bohn's nodules, alveolar cysts, palatal cyst, gingival cyst, ankyloglossia, Fordyce's spots/granules, and natal/neonatal teeth. ${ }^{5}$

Alveolar cysts of the newborn are common lesions, reported in up to one-half of all newborns, presenting as individual cysts no more than 2 to $3 \mathrm{~mm}$ in diameter. ${ }^{6}$ Alveolar cysts are the remnants of degenerating dental lamina found on the buccal, lingual, or crest portion of the alveolar ridge. ${ }^{7}$ The reported prevalence of alveolar cysts in newborn ranges from $25 \%$ to $53 \% .^{8}$ They are more common

\footnotetext{
${ }^{1-4}$ Department of Pediatrics and Preventive Dentistry, Subharti Dental College and Hospital, Swami Vivekanand Subharti University, Meerut, Uttar Pradesh, India

Corresponding Author: Madan M Niranjan, Department of Pediatrics and Preventive Dentistry, Subharti Dental College and Hospital, Swami Vivekanand Subharti University, Meerut, Uttar Pradesh, India, Phone: +919412015029, e-mail: madanniranjan639@gmail.com

How to cite this article: Niranjan MM, Srivastava N, Rana V, et al. Assessment of Intraoral Findings of Neonates, Born in and around Meerut City. Int J Clin Pediatr Dent 2020;13(1):48-52.

Source of support: Nil

Conflict of interest: None
}

in the maxilla than the mandible. The prognosis is good and no treatment is required.

Palatal cysts of the newborn are small 1-3 mm, white or yellow papule found mostly along the midline or near the junction of the hard and soft palate thought to be originated from the epithelial cells that persist at the site of fusion of the palatal shelves. ${ }^{7}$ The prevalence of palatal cysts is about $60 \%$ in infants. They are frequently clustered in groups of 2 to 6 cysts. They are self-limiting so no treatment is required. Dental lamina cysts, also known as gingival cysts of newborns, are a benign and asymptomatic oral mucosal lesion found on the alveolar ridge of newborns or very young infants that represent cysts originating from remnants of the dental lamina. ${ }^{5}$ Dental lamina cysts occur as white or pink small nodules, often multiple, approximately 1 to $3 \mathrm{~mm}$ in diameter located on the midpalatine raphe and less frequently on the maxillary and mandibular alveolar mucosa. 
Epstein's pearls are keratin-filled cysts that occur in the mid palatine raphe region near the mucosal surface. The prevalence of Epstein's pearls is $35.2 \%$ with no sexual predilection. ${ }^{8}$ These are believed to arise from the trapped embryological epithelial remnants present along the line of fusion of the palatal halves. On the other hand, Bohn's nodules, originally described in 1866 as remnants of mucous gland tissue, are found on the buccal or lingual aspects of the dental ridges. Bohn's nodules are also keratin-filled cysts but scattered over the palate, most numerous along the junction of the hard and the soft palate and are derived from the palatal salivary gland structure. Bohn's nodules are seen in $47.4 \%$ of infants and newborns with no sex predilection. ${ }^{4}$ Although the prevalence is high, these cysts are rarely seen by the dentist or pediatrician because of the transient nature of these cysts, which disappear within 2 weeks to 5 months of postnatal life.

Fordyce's spots, also known as Fordyce's glands, increase with age, being more common in adults than in children with a male to female ratio of approximately 2:1. Fordyce's spots are enlarged sebaceous glands that can be seen on various body parts such as the lips, oral mucosa, penis, and labia. ${ }^{9}$

Ankyloglossia is a developmental anomaly of the tongue seen in newborns. Reported to vary from $0.4 \%$ to $4.8 \%$ with a predilection for males to females as from $5: 1$ to $4: 1 .^{10}$ It is characterized by a short, thick lingual frenum resulting in the limitation of tongue movement leading to problems with breastfeeding, licking lips, using the tongue to sweep the teeth free of debris and speech articulation. Certain therapies like frenotomy or frenuloplasty may be performed to treat it. ${ }^{10}$

Another oral anomaly of interest is natal and neonatal teeth. The term "natal teeth" is defined as the presence of teeth at birth and neonatal teeth present within 30 days of birth. Most natal teeth are normal primary dentition (95\%), while a small percentage is supernumerary. A majority (85\%) of the natal teeth are mandibular incisors. ${ }^{11}$ If the natal teeth are firm and asymptomatic, conservative management is indicated.

Most of the studies, which describe abnormal and normal findings of neonates, were from the western population and there are very few studies conducted in the Indian population. Considering the above-mentioned factors, the present study was planned to record intraoral findings of neonates in and around Meerut, India.

\section{Materials and Methods}

This study was conducted at the Department of Paedodontics and Preventive Dentistry, Subharti Dental College, Meerut, in collaboration with the Department of Gynecology, Subharti Medical College and Hospital, Meerut, India. A sample size of 1,000 infants from birth to 1 month of age was examined. The approval from the Institutional Ethical Committee was obtained prior to the start of the study. Written informed consent was obtained from the parents of the infants.

The examination of the oral cavity was performed with the aid of disposable tongue depressors, sterile mouth mirrors, and torchlight. Sterile latex gloves were used to inspect the oral cavity, with an index finger. The antero-posterior and buccolingual relationships of the alveolar ridges were assessed carefully by bringing the jaws together and other intraoral findings were recorded.

The following findings were recorded:

Oral mucosal cysts: whitish changes of macula or papule-like appearance, with clearly defined borders on the alveolar ridge (gingival cyst) or palate (palatal cyst) (Fig. 1A).
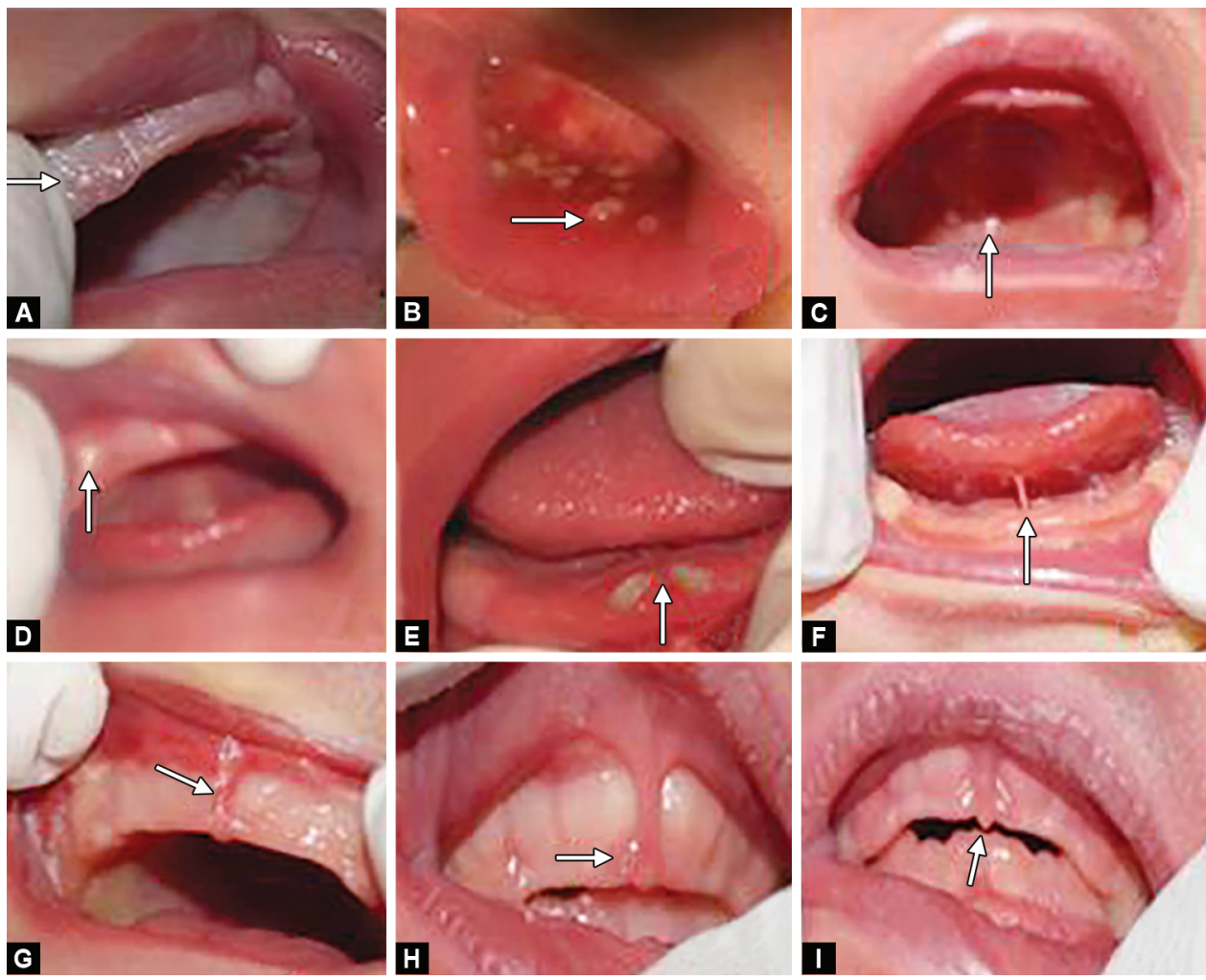

Figs 1 A to l: (A) Oral mucosal cyst; (B) Fordyce spot; (C) Epstein Pearls; (D) Bohn's nodule; (E) Natal or neonatal teeth; (F) Ankyloglossia; (G) Attachment of upper midline frenum; $(\mathrm{H})$ Buccolingual relationship; (I) Open bite 


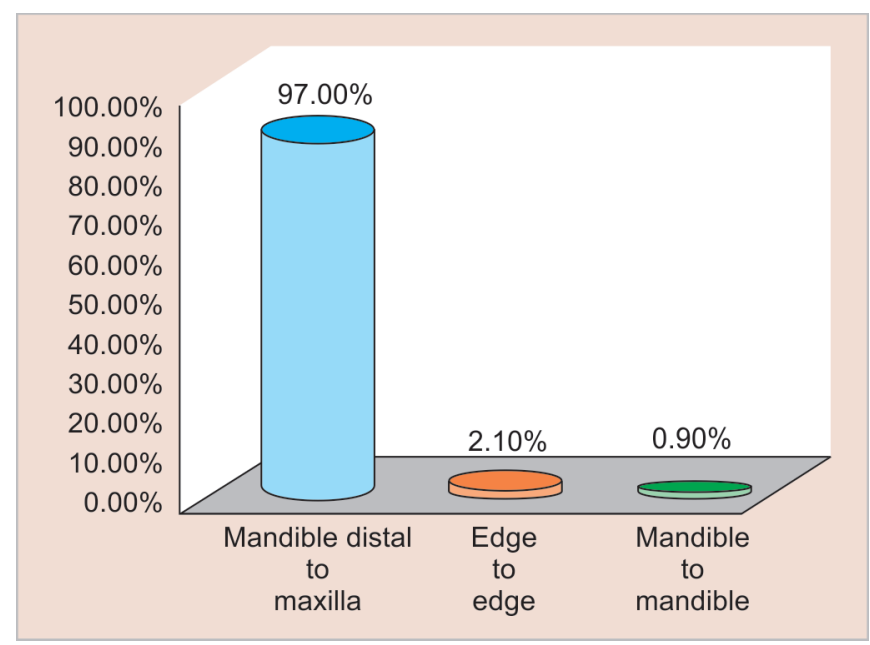

Fig. 2: Antero-posterior relationship between alveolar ridges (maxillary and mandibular)

Table 1: Antero-posterior relationship of mandible to maxilla

\begin{tabular}{lll}
\hline Antero-posterior relationship & Number & Percentage \\
\hline Mandible distal to maxilla & 970 & 97.0 \\
Edge-to-edge & 21 & 2.1 \\
Mandible mesial to maxilla & 9 & 0.9 \\
Total & 1,000 & 100.0 \\
\hline
\end{tabular}

Fordyce's spot: Minute yellowish papules on the buccal mucosa, with a clearly defined border to the surrounding tissue (Fig. 1B).

Epstein's pearls: Keratin-filled cysts that occur in the mid palatine raphe region near the mucosal surface and are believed to arise from the trapped embryological epithelial remnants present along the line of fusion of the palatal halves (Fig. 1C).

Bohn's nodules: Heterotopic salivary gland remnants located on the buccal or lingual surface of the alveolar ridge (not the crest), or on the hard palate, away from the raphe (Fig. 1D).

Natal or neonatal teeth: the presence of the incisal edge of a tooth which penetrates the mucous membrane (Fig. 1E).

Ankyloglossia: diagnosed if the lingual frenum is attached close to the border of the papillated part of the tongue, preventing protrusion of the tongue (Fig. 1F).

Attachment of the upper midline frenum was recorded as being either at the crest, or buccally or palatally on the alveolar ridge (Fig. 1G).

The relationship between the alveolar ridges was recorded. The antero-posterior relationship was classified as distal, edge-toedge, or mesial position of the anterior segment of the mandibular ridge in relation to the maxillary ridge. The buccolingual relationship was classified as a lingual, edge-to-edge, or buccal position of the mandibular ridges in relation to the maxillary arch (Fig. 1H).

The open bite was recorded when there was a vertical space between the anterior segments of the maxillary and mandibular alveolar ridges when these were brought together (Fig. 11). However, it could not be measured.

The location of intraoral findings was also recorded and photographs of representative cases were taken.

The statistical analyses used in the study were arithmetic means and standard deviations, which were utilized using SPSS software version 21.0 and Epi-info version 3.0.

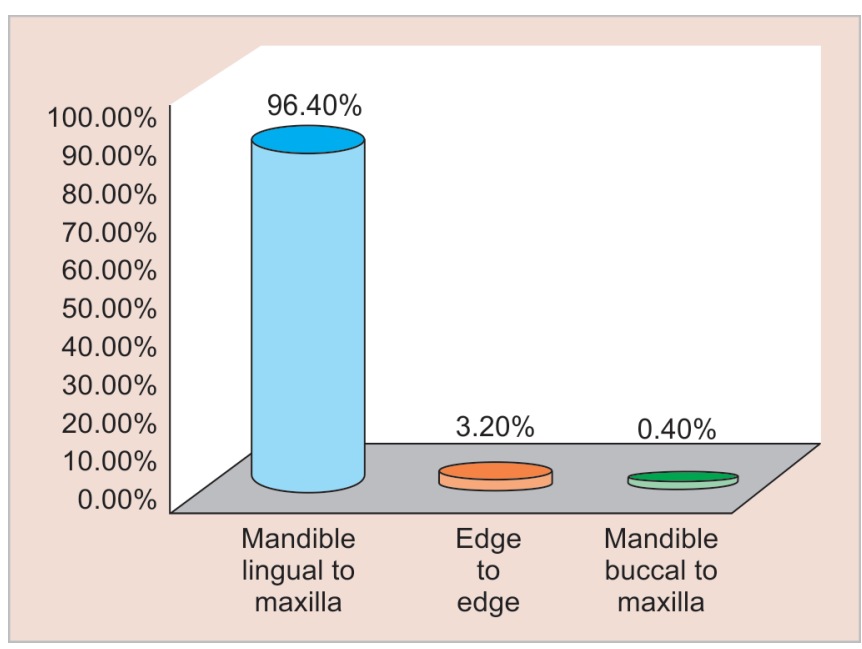

Fig. 3: Buccolingual relationship between alveolar ridges (maxillary and mandibular)

Table 2: Buccolingual relationship of mandible to maxilla

\begin{tabular}{lll}
\hline Buccolingual relationship & Number & Percentage \\
\hline Mandible lingual to maxilla & 964 & 96.4 \\
Edge-to-edge & 32 & 3.2 \\
Mandible buccal to maxilla & 4 & 0.4 \\
Total & 1,000 & 100.0 \\
\hline
\end{tabular}

Table 3: Variation in location of attachment of maxillary midline labial frenum

\begin{tabular}{lll}
\hline Extent & Number & Percentage \\
\hline On the buccal aspect of alveolar ridge & 88 & 8.8 \\
Crest of alveolar ridge & 791 & 79.1 \\
Extending to palatal to alveolar crest & 121 & 12.1 \\
Total & 1,000 & 100.0 \\
\hline
\end{tabular}

\section{Results}

The mean age of mothers (in years) was $426.63 \pm 1.63$. The minimum age among the study population was 18 years and the maximum age among the study population was 35 years. The mean age of infant was $6.11 \pm 1.35$ days that is the minimum age was 1 day and the maximum age among the study population was 25 days.

A comparison of the vertical relationship of gum pads among the newborn, 91 infants (9.1\%) showed an anterior open bite while remaining 909 (90.9\%) were normal.

In the antero-posterior relationship, the most common relationship of mandible was distal to maxilla (97.0\%). The edge-toedge relationship was present in 21 infants (2.1\%). In nine infants, maxilla was present mesial to mandible (0.9\%) (Table 1 and Fig. 2).

In the buccolingual relationship, the most common relationship of mandible was lingual to maxilla (96.4\%) followed by the edgeto-edge relationship (3.2\%). The least common relationship was buccal to maxilla (0.4\%) (Table 2 and Fig. 3 ).

The maxillary midline labial frenum showed a variation in its location such as the attachment of labial frenum at the buccal of the alveolar ridge was present in 88 infants (8.8\%), at crest of alveolar ridge in 791 infants (79.1\%) and palatal to alveolar ridge in 121 infants (12.1\%) (Table 3 and Fig. 4). 


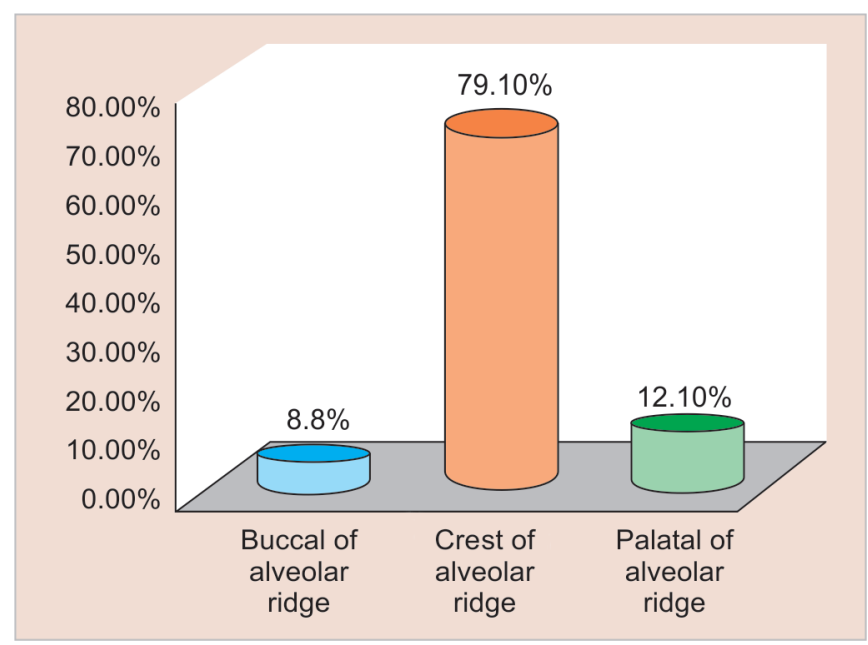

Fig. 4: Variation in location of attachment of maxillary midline labial frenum

Natal/neonatal teeth were found in only six infants ( $0.6 \%)$. The study population showed that the ankyloglossia was present among five $(0.5 \%)$ infants only. On intraoral examination, Bohn's nodules were present in 388 (38.8\%) infants among the study population. On intraoral assessment, the distribution of Epstein's pearls was assessed in 383 (38.3\%) infants among the population. Oral mucosal cysts were found in $61.2 \%$ of infants. The great majority of these were present in the maxilla (86\%) (Fig. 5).

\section{Discussion}

In the present study, the oral mucosal cysts were present in $61.2 \%$ of the infants. About $86 \%$ of the infants showed the presence of cysts in the maxillary region and $14 \%$ in the mandibular region whereas Fromm in 1967 reported a high prevalence of oral mucosal cysts in $75.9 \% .{ }^{12}$ Cataldo and Berkman reported that the most common site of the oral mucosal cyst was the maxillary region (65.1\%) and the least common site was the mandibular alveolar mucosa $(9.9 \%) .{ }^{13}$

George et al. examined 1,038 newborn Indian babies and found that gingival cysts were present in $13.8 \%$ newborn, $10.8 \%$ on the alveolar region, $1.8 \%$ palatally, and less than $1 \%$ was present on the mandibular ridge. ${ }^{2}$ Paula et al. reported $28 \%$ of prevalence of oral mucosal cyst. ${ }^{14}$ Friend et al. recorded the alveolar cyst in $25 \%$ of children from Tennessee, USA. ${ }^{15}$ In the present study, the researched data were in contrast with the above studies.

In the present study, $38.3 \%$ of Epstein pearls were located in the midline within the median raphae of the hard palate in the maxilla, which were similar to the study by George et al, i.e., $35.2 \%{ }^{2}$

In the present study, $38.8 \%$ of Bohn's nodules were located at the junction of the hard and soft palate, adjacent to the mid-palatal raphae which were almost similar to the prevalence showed by George et al. in 2008 in a study of 492 newborns where Bohn's nodules of $47.4 \%$ were found. ${ }^{2}$

In the present study, Fordyce's spots found on the buccal mucosa in only $1.2 \%$ of cases which were similar to the study done by the Flinck et al., i.e., $1 \%$ in $1994 .^{3}$

The incidence of natal/neonatal teeth varies from 1:800 to $1: 6,000$ livebirths. Females have a greater predilection. Natal teeth are frequently seen in the anterior mandible (85\%) and anterior maxilla (11\%). A majority (85\%) of natal teeth are mandibular incisors. ${ }^{16}$
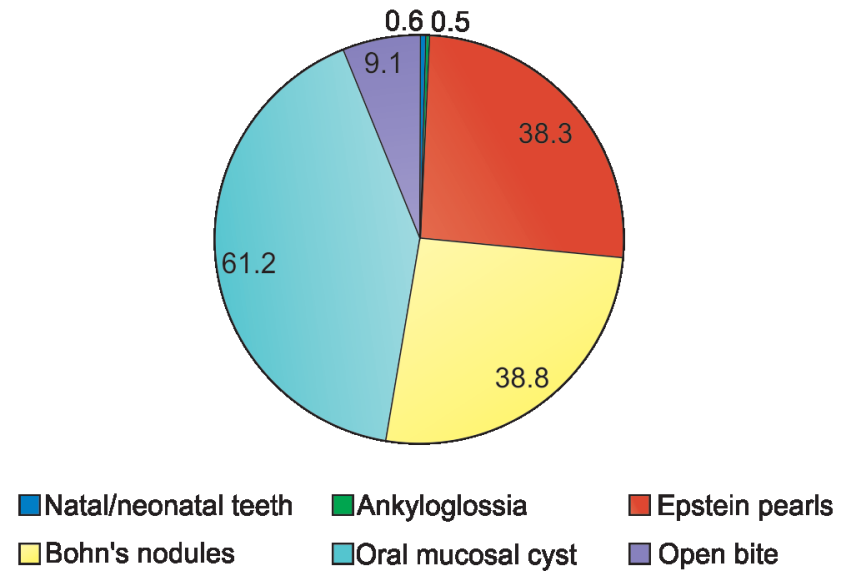

Fig. 5: Distribution of various anomalies of infants in study population

In our study, only $0.6 \%$ of infants were found with natal teeth. Kates et al. ${ }^{17}$ reported that prevalence of natal/neonatal teeth was $0.07 \%$; King and Lee, ${ }^{18}$ Liu et al., ${ }^{19}$ and Friend et al. ${ }^{15}$ also reported the prevalence of natal/neonatal teeth as $0.07 \%, 0.09 \%$ and 0.7 , which was nearly similar to the results of the present study.

In the present study, the maxillary midline labial frenum showed a variation in its location as the attachment of labial frenum at the buccal of the alveolar ridge was present in $8.8 \%$, at the crest of the alveolar ridge in $79.1 \%$ in the palatal to the alveolar ridge in $12.1 \%$ of cases.

A study conducted by Flinck et al. showed the maxillary labial frenum to be located buccal to the alveolar ridge in $6.7 \%$ of the cases, at the crest of the alveolar ridge in $76.7 \%$ and the palatal to the alveolar ridge in $16.7 \%{ }^{3}$ Ranly in 1998 has also stated that the labial frenum may be continuous with the incisive papilla in $25 \%$ of newborns. ${ }^{20}$

The frequency of ankyloglossia has been reported to vary from $0.4 \%$ to $4.8 \%$ with a predilection for males to females from $1.5: 1$ to $4: 1 .^{10}$ In the present study, ankyloglossia was present among $0.5 \%$ of infants which were similar to the study done by George et al. $(0.5 \%){ }^{2}$ Our results of prevalence of ankyloglossia were not in accordance with the above studies.

In the present study, the anterior open bite was present in 9.1\% of the infants. Flinck et al. found the anterior open bite in $39.8 \%$ of the newborns ${ }^{3}$ which was much lower than that reported by Clinch (62.5\%); however, Simpson and Cheung reported only $1.33 \%$ of infants with an open bite in the Canadian population.

In our study, $97 \%$ of neonates showed the antero-posterior relationship of gum pads as mandible distal to maxilla, $2.1 \%$ edgeto-edge, and only $0.9 \%$ maxilla mesial to mandible; these findings were similar to the findings of Flinck et al. ${ }^{3}$ who reported that $99 \%$ of the cases had mandible distal to maxilla, ${ }^{3}$ while only $1 \%$ had edge-to-edge.

In the present study, $96.4 \%$ of neonates showed the buccolingual relationship of gum pads as mandible lingual to the maxilla, $3.2 \%$ edge-to-edge, and only $0.4 \%$ mandible buccal to maxilla. Flinck et al. reported similar findings as $97.6 \%$ lingual position of the mandible relative to maxilla, edge-to-edge in $2.2 \%$, and mandible buccal to maxilla only in $0.2 \%$ of cases. ${ }^{3}$

The results of the present study confirmed the prevalence of intraoral findings of newborns in earlier studies that oral mucosal cysts are the most common findings. Knowledge of these frequently found lesions in infants is, therefore, crucial for all healthcare 
providers, including dental surgeons and pediatricians, who may be contacted by the parents.

In many situations, no treatment is required for lesions such as Fordyce's granules, mucosal cysts, and infants are kept under observation but sometimes early interventions are required in the cases of ankyloglossia and natal teeth so that quality of life of a child can be improved.

\section{Conclusion}

Based on the results of the present study, the following conclusions were drawn:

- The present study confirmed the presence of the anterior open bite only in $9.1 \%$ of cases, while this percentage has been reported to be quite high in already existing literature (approximately 38\%).

- When the maxillo-mandibular relationship of gum pads was examined, it was found that in approximately $97 \%$ cases, the mandible was distal and lingual to maxilla.

- The most common intraoral finding in examined neonates was an oral mucosal cyst (61.2\%). These cysts were mostly found in maxilla (86\%).

- The presence of Epstein's pearls was the next most common findings found in $38.3 \%$ of neonates.

- Fordyce's spots were found only in $1.2 \%$ cases and were not a very common finding.

- Only 6 out of 1,000 neonates examined had the presence of natal/neonatal teeth $(0.6 \%)$. The most common site being anterior mandible.

- Ankyloglossia was also an uncommon finding and was present in only $0.5 \%$ of neonates.

- When maxillary frenum was examined, it was found that in $79.1 \%$ of cases, it was attached on the alveolar crest, while in $12.1 \%$, it was palatal to the alveolar crest. About $8.8 \%$ of neonates had their frenum attached on the buccal slope of the alveolar ridge.

\section{References}

1. Marini R, Chipaila N, et al. Unusual symptomatic inclusion cysts in a newborn: a case report. J Med Case Rep 2014;8:314. DOI: 10.1186/17521947-8-314.
2. George D, Bhat SS, et al. Oral findings in newborn children in and around Mangalore, Karnataka State, India. Med Princ Pract 2008;17(5):385-389. DOI: 10.1159/000141502.

3. Flinck $A$, Paludan $A$, et al. Oral finding in a group of newborn Swedish children. Int J Paediatr Dent 1994;4:67-73. DOI: 10.1111/j.1365263X.1994.tb00107.x.

4. Freudenberger S, Santos Diaz MA, et al. Intraoral findings and other developmental conditions in Mexican neonates. J Dent Child 2008;75:280-286.

5. Benni DB, Sirur S. Gingival cyst of the newborn: a case report. Int Dent African Ed 2000;3(2):32-34.

6. Neville BW, Damm DD, et al. Oral and Maxillofacial Pathology, 3rd edn, Saunders, 2009.

7. Vasanthakumari A, Shanmugavadivel G, et al. Sch J Dent Sci 2016;3:7173.

8. Sahu S, Maurya R, et al. Multiple congenital epulis in newborn-a rare presentation. J Oral Maxillofac Pathol 2009;13(2):78-80. DOI: 10.4103/0973-029X.57674.

9. Leung AKC, Barankin B. Fordyce Spots. Clin Case Rep Rev 2015;1(6):121122.

10. Messner AH, Lalakea ML. Ankyloglossia: controversies in management. Int J Pediatr Otorhinolaryngol 2000;54(2-3):123-131. DOI: 10.1001/ archotol.126.1.36.

11. Cunha RF, Boer FA, et al. Natal and neonatal teeth. Pediatr Dent 2001;23(2):158-162.

12. Fromm A. Epstein's pearls, Bohn's nodules, and inclusion cyst of the oral cavity. J Dent Child 1967;24:275-87.

13. Cataldo E, Berkman MD. Cysts of the oral mucosa in newborns. Am J Dis Child 1968;116:44. DOI: 10.1001/archpedi.1968.02100020046006.

14. Paula JDR, Dezan CC, et al. Oral and facial inclusion cysts in newborns. J Clin Pediatr Dent 2006;31(2):127-129. DOI: 10.17796/ jcpd.31.2.rw3h853m3rk242q0.

15. Friend GW, Harris EF, et al. Oral anomalies in the neonate, by race and gender, in an urban setting. Pediatr Dent 1990;12(3):157-161.

16. Zhu J, King D. Natal and neonatal teeth. ASDC J Dent Child 1995;62:123-128.

17. Kates $\mathrm{GA}$, Needleman NL, et al. Natal and neonatal teeth: a clinical study. J Am Dent Assoc 1984;109:441-443. DOI: 10.14219/jada. archive.1984.0415.

18. King NM, Lee AM. Prematurely erupted teeth in newborn infants. J Pediatr 1989;109:441-443. DOI: 10.1016/S0022-3476(89)80142-8.

19. Liu MH, Huang WH. Oral Abnormalities in Taiwanese Newborns. J Dent Child 2004;71:118-120.

20. Ranly DM. Early orofacial development. J Clin Pediatr Dent 1998;22(4):267-275. 